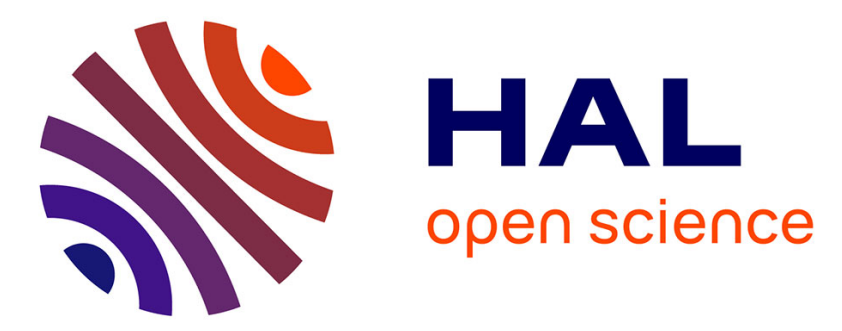

\title{
Stress perçu et santé physique des doctorants dans les universités françaises
}

P. Haag, R. Shankland, E. Osin, É. Boujut, Fabienne Cazalis, A.-S. Bruno, P. Vrignaud, M.-C. Gay

\section{To cite this version:}

P. Haag, R. Shankland, E. Osin, É. Boujut, Fabienne Cazalis, et al.. Stress perçu et santé physique des doctorants dans les universités françaises. Pratiques Psychologiques, 2018, 24 (1), pp.1-20. 10.1016/j.prps.2017.04.005 . hal-02424380

\section{HAL Id: hal-02424380 \\ https://hal.science/hal-02424380}

Submitted on 1 Mar 2021

HAL is a multi-disciplinary open access archive for the deposit and dissemination of scientific research documents, whether they are published or not. The documents may come from teaching and research institutions in France or abroad, or from public or private research centers.
L'archive ouverte pluridisciplinaire HAL, est destinée au dépôt et à la diffusion de documents scientifiques de niveau recherche, publiés ou non, émanant des établissements d'enseignement et de recherche français ou étrangers, des laboratoires publics ou privés. 


\title{
Stress perçu et santé physique des doctorants dans les universités françaises
}

\author{
Perceived stress and physical health among French university \\ PhD students \\ P. Haag ${ }^{\mathrm{a}, \mathrm{b}, *}$, R. Shankland ${ }^{\mathrm{d}}$, E. Osin ${ }^{\mathrm{c}}$, É. Boujut $^{\mathrm{g}}$, F. Cazalis ${ }^{\mathrm{e}}$, \\ A.-S. Bruno ${ }^{\mathrm{f}}$, P. Vrignaud ${ }^{\mathrm{a}}$, M.-C. Gay ${ }^{\mathrm{a}}$ \\ a Département de psychologie, EA 4430, évaluation clinique des troubles psychopathologiques et des psychothérapies, \\ université Paris Ouest Nanterre La Défense, 200, avenue de la République, 92000 Nanterre, France \\ b Institut de recherches interdisciplinaires sur les enjeux sociaux-sciences sociales, politique, santé, UMR 8156, École \\ des hautes études en sciences sociales, 54, boulevard Raspail, 75006 Paris, France \\ c Psychology department, National Research University Higher School of Economics, 20, Myasnitskaya il, 101000 \\ Moscou, Russie \\ d Laboratoire interuniversitaire de psychologie personnalité, cognition, changement social, EA 4145, université \\ Pierre-Mendès, 1251, avenue Centrale, 38040 Grenoble, France \\ e Institut Jean-Nicod, UMR 8129, École normale supérieure, 29, rue d'Ulm, 75005 Paris, France \\ ${ }^{\mathrm{f}}$ Centre d'histoire sociale du XX ${ }^{e}$ siècle, UMR 8058, université Paris 1, Panthéon-Sorbonne, 9, rue Malher, 75004 \\ Paris, France \\ ${ }^{g}$ Laboratoire de psychopathologie et processus de santé, EA 4057, université Paris-Descartes, 71, avenue \\ Édouard-Vaillant, 92100 Boulogne-Billancourt, France
}

\section{Résumé}

Alors que la santé physique et mentale des étudiants de premier cycle universitaire a fait l'objet de nombreuses recherches, y compris en France, plus rares sont les publications portant sur les effets du stress chez les doctorants. La présente étude, réalisée auprès d'un large échantillon de doctorants $(n=2013)$, a mesuré l'effet de variables socio-démographiques et de comportements de santé sur le stress perçu et les symptômes somatiques. Les résultats montrent que le niveau de stress perçu des doctorants est élevé. Les prédicteurs du stress sont les suivants : sexe, âge, filière, satisfaction liée à l'encadrement, activité physique

* Auteur correspondant.

Adresse e-mail : ph@ehess.fr (P. Haag). 
et sommeil. Dans une perspective transactionnelle, cette recherche apporte des éléments significatifs de compréhension d'une population encore peu étudiée et propose des pistes de recherche et d'application.

Mots clés : Santé ; Enseignement supérieur ; Stress perçu ; Sommeil ; Activité physique

\begin{abstract}
Few studies have focused on $\mathrm{PhD}$ students in France despite the fact that this population appears to be particularly vulnerable to stress and often faces precariousness. This research investigated associations between socio-demographic factors, health behaviours, perceived stress and physical health in a wide sample of PhD students $(n=1923)$ in order to have a better understanding of this population's specificities. Our results indicated that stress is a critical issue among $\mathrm{PhD}$ candidates. Predictors of perceived stress were: gender, age, satisfaction with supervision, sleep quality and sports practice. Findings from this study confirm the relevance of the transactional model to investigate students' health issues and suggest that physical activity and stress management should be encouraged through health promotion programs.
\end{abstract}

Keywords: Health; Higher education; Perceived stress; Sleep quality; Physical activity

\title{
1. Introduction
}

Plusieurs enquêtes récentes se sont intéressées à la qualité de vie et à la santé physique ou mentale d'étudiants de premier cycle universitaire en France et ont montré que cette dernière était généralement préoccupante en raison de la prévalence de dépression, de troubles du sommeil et d'idées suicidaires (Boudoukha, Bonnaud-Antignac, Acier, \& Chaillou, 2011 ; Boujut, Koleck, Bruchon-Schweitzer, \& Bourgeois, 2009 ; Hammoud, 2005 ; Ladner, Mihailescu, Kern, Romo, \& Tavolacci, 2016 ; Mazé \& Verlhiac, 2013 ; Migeot et al., 2006 ; Saleh, Camart, \& Romo, 2017). En revanche, les études afférentes à la santé physique et mentale des jeunes chercheurs en fin de cursus universitaire que sont les doctorants sont peu nombreuses et inexistantes en France. Faire une thèse constitue pourtant une expérience souvent éprouvante, tant sur le plan universitaire que personnel. Le stress et toutes sortes d'émotions négatives constituent des « descripteurs » récurrents de l'expérience des doctorants (Aspland, Edwards, O'Leary, \& Ryan, 1999 ; Austin, 2002 ; Hawley, 1993 ; Lovitts \& Nelson, 2000 ; Nyquist et al., 1999). Certains auteurs vont jusqu'à affirmer que le stress est au cœur de l'expérience de l'étudiant de $3^{\mathrm{e}}$ cycle (Offstein, Larson, McNeill, \& Mwale, 2004). Enfin, le fait que la condition de doctorant elle-même soit censée être pénible et associée à une diminution significative du bien-être est admis de longue date par l'ensemble des acteurs (Golde, 1998 ; Hawley, 1993 ; Kurtz-Costes, Helmke, \& Ülkü-Steiner, 2006 ; Lovitts \& Nelson, 2000 ; Nyquist et al., 1999 ; Vekkaila, Pyhältö, \& Lonka, 2013). Dans un contexte où le nombre de doctorats délivrés est en progression constante (Gérard, 2014 ; RERS, 2014, indicateur 8,20), cette recherche vise à mieux connaître les spécificités de cette population sur le plan du stress et de la santé dans notre pays.

Selon le modèle transactionnel proposé par Lazarus et Folkman (1984), le stress est la relation particulière entre la personne et l'environnement, évaluée par la personne comme dépassant ou excédant ses ressources et mettant en danger son bien-être. Il ne réside donc ni uniquement 
dans l'événement, ni uniquement dans l'individu, mais dans la transaction entre ce dernier et son environnement. La dimension subjective de ce processus est centrale ici, car deux personnes peuvent réagir différemment à un même stresseur. Il est donc nécessaire d'opérer une distinction entre les stresseurs environnementaux objectifs et leur impact subjectif dans une situation donnée (Bruchon-Schweitzer, 2002). C'est par ce dernier que se caractérise le stress perçu. Une multitude de facteurs - contextuels et personnels - influence la perception d'un événement comme une menace ou comme un défi. Le stress apparaît comme un mode de traitement transitoire, spécifique à une situation.

Dans un premier temps nous allons présenter les données concernant le stress et la santé des étudiants en général, et des doctorants en particulier, de même que les répercussions du stress sur la santé.

\subsection{Stress et santé dans l'enseignement supérieur}

Les liens entre stress et santé sont aujourd'hui attestés par un important corpus de recherches, qui s'accordent sur le fait que différents facteurs psychobiologiques ou comportementaux sont susceptibles d'affecter cette relation (Lovallo, 2005). À un niveau élevé de stress sont associés toutes sortes de manifestations physiques telles que diabète, insomnie, céphalées, maladies cardiovasculaires, maladies auto-immunes (Cherewatenko \& Perry, 2003). Le stress affecte aussi bien la santé physique (Lovell, Moss, \& Wetherell, 2011) que le bien-être ou la qualité de vie (Sjögren, Leanderson, Kristenson, \& Ernerudh, 2006).

Il est établi que l'activité physique contribue à réduire le stress et qu'elle favorise l'amélioration des symptômes somatiques (Boujut \& Décamps, 2012 ; Tavolacci et al., 2013 ; Taylor, 2000). L'arrivée à l'université s'accompagne fréquemment de changements d'habitudes de vie, en particulier d'une diminution significative de l'activité physique (Boujut \& Décamps, 2012 ; Dinger, 1999 ; Longfield, Romas, \& Irwin, 2006 ; Reynolds, 2007). Ceci a pour effet d'augmenter le stress. En effet, les étudiants sportifs reportent moins de stress perçu et de symptômes psychologiques que les non sportifs (Bhui, 2002 ; Nguyen-Michel, Unger, Hamilton \& Spruijt-Metz, 2006 ; Skirka, 2000) et l'efficacité d'une activité physique, même modérée, sur la santé est attestée (Bouchard, Blair, \& Haskell, 2012 ; Powell, Paluch, \& Blair, 2011). De plus, la qualité du sommeil est également corrélée à un moindre degré de stress.

Le stress psychosocial et le stress physiologique sont tous deux corrélés aux variations de sommeil (Abdulghani et al., 2012; Mezick et al., 2009). Le manque de sommeil est un problème croissant dans les sociétés occidentales, en particulier chez les femmes, et accentue la vulnérabilité au stress (Wright, Valdimarsdottir, Erblich, \& Bovbjerg, 2007). Chez les étudiants, le stress académique est associé à une mauvaise qualité de sommeil (Lund, Reiter, Whiting, \& Prichard, 2010 ; Pallos, Gergely, Yamada, Miyazaki, \& Okawa, 2007 ; Winwood \& Lushington, 2006). Cette relation entre les troubles du sommeil et le stress apparaît comme bidirectionnelle (Brand et al., 2010), de même que la relation entre le sommeil et l'activité physique (Kline, 2014).

L'une des variables les plus reconnues comme ayant un effet modérateur sur le stress est le genre : les femmes présentent une plus grande vulnérabilité au stress que les hommes (Alonso et al., 2004 ; Davis, Burleson, \& Kruszewski, 2011). Cette différence est observée de façon récurrente en population étudiante, dès le lycée (Zakari, Walburg, \& Chabrol, 2008), et sous diverses latitudes - par exemple Royaume-Uni, États-Unis, Chine (Flynn, Schipper, Roach, \& Segerstrom, 2009 ; Liu et al., 2007 ; McCleary \& Zucker, 1991 ; Saklofske, Austin, Mastoras, Beaton, \& Osborne, 2012 ; Zascavage, Winterman, Buot, Wies, \& Lyzinski, 2012). 
Ce constat vaut également pour les étudiants de $3^{\mathrm{e}}$ cycle (Bireda, 2015 ; Oswalt \& Riddock, 2007).

\subsection{Le stress chez les doctorants : un sujet encore peu étudié}

Bien que les études publiées à ce jour concernent majoritairement des étudiants de premier cycle universitaire, un nombre croissant de chercheurs s'intéresse aujourd'hui à cette population. Toutefois, comme l'indiquent El-Ghoroury, Galper, Sawaqdeh, et Bufka (2012) et McKinzie, Altamura, Burgoon, et Bishop (2006), les recherches sur le stress et les stratégies d'ajustement chez les étudiants de $3^{\mathrm{e}}$ cycle sont encore limitées et hétérogènes ; de plus, elles consistent encore essentiellement de mémoires et de thèses non publiés (p. ex. Armstrong, 2014 ; Benesek, 1998 ; Hill, 2011 ; Peters, 2007 ; Wulf, 2010). Ces dernières concernent d'ailleurs le plus souvent des doctorants en psychologie et, de manière générale, les professions de santé restent de loin les plus étudiées (p. ex. Aktekin et al., 2001 ; Sasidharan, Kolasani, \& Divyashanti, 2016 ; Shah, Bazargan-Hejazi, Lindstrom, \& Wolf, 2009). Les auteurs justifient le choix de cette population par l'importante responsabilité qui pèse sur les épaules des jeunes professionnels engagés dans une relation d'aide et chargés d'accompagner des personnes en souffrance. En effet, stress et épuisement professionnel peuvent les amener à prendre de mauvaises décisions sur le plan clinique, voire à adopter des comportements dangereux ou qui s'écartent de l'éthique (Barnett, Baker, Elman, \& Schoener, 2007 ; El-Ghoroury et al., 2012 ; Elman \& Forrest, 2007). Mais les données concernant d'autres filières font cruellement défaut. En outre, même lorsque les observations concernent les étudiants d'autres disciplines que les professions de santé (Grady, La Touche, Oslawski-Lopez, Powers, \& Simacek, 2014 ; Helmers, Danoff, Steinert, Young, \& Leyton, 1997 ; Johnson, Batia, \& Haun, 2008 ; Oswalt \& Riddock, 2007), les participants sont le plus souvent inscrits dans des universités nord-américaines. Alors qu'il est établi que l'expérience de la thèse varie considérablement d'un pays à l'autre (Matos, 2013). Puisque les données concernant les doctorants en dehors du champ de la santé et dans des aires géographiques autres que l'Amérique du Nord sont encore lacunaires, il est important de diversifier le champ des investigations (Knox et al., 2011 ; Schlosser \& Gelso, 2001).

Les études déjà réalisées présentent d'autres lacunes que le présent travail se propose de contribuer à combler. Dans les études nord-américaines, la distinction entre les niveaux de master et de doctorat n'est pas toujours explicite : nombre d'études portent sur les étudiants de $3^{\mathrm{e}}$ cycle dans leur ensemble (graduate students). Or les aspirations et les besoins des étudiants de master ne sont pas identiques à ceux des doctorants, de même que les attentes de l'université à leur égard. Il est donc important de les différencier. De plus, l'approche qualitative et les analyses fondées sur des entretiens ou sur une observation ethnographique sont largement majoritaires. Les études quantitatives utilisant des questionnaires validés dans le champ de la psychologie sont moins fréquentes, ce qui pose un problème quant à la généralisation des résultats. Enfin, les grandes orientations des recherches actuelles sur les doctorants concernent principalement l'abandon en cours de thèse, la relation avec le directeur de recherche, l'adaptation aux mutations actuelles de l'univers de l'enseignement supérieur et les pratiques académiques (Matos, 2013 ; Trafford \& Leshem, 2009). La santé physique et mentale des doctorants a donné lieu a un nombre limité de travaux, que nous évoquons maintenant. Les études quantitatives portent sur les étudiants de $3^{\mathrm{e}}$ cycle (mais pas nécessairement sur les seuls doctorants) établissent un lien entre l'avancée dans le cursus universitaire et le niveau d'anxiété, de stress ou de dépression (Armstrong, 2014 ; 
Nogueira-Martins, Neto, Macedo, Citero, \& Mari, 2004 ; Stecker, 2004). Cette dernière s'accompagne également d'une prévalence croissante d'idéations suicidaires (Fogg, 2009). La santé physique est plus rarement évoquée, malgré quelques exceptions (Schmidt \& Umans, 2014 ; Wulf, 2010).

Notre étude se propose d'apporter de nouvelles données, quantitatives, centrées sur la population des doctorants de diverses filières, dans une aire géographique où ils sont encore peu étudiés et en s'intéressant aux liens entre stress et santé.

\subsection{Choix du cadre théorique de référence}

Compte tenu de l'hétérogénéité des déterminants qui interviennent dans cette phase ultime du parcours universitaire, le modèle transactionnel, intégratif et multifactoriel élaboré par BruchonSchweitzer (2002) nous a semblé particulièrement pertinent pour aborder la question de la santé des doctorants. Ce modèle s'articule autour de trois composantes : les antécédents, les processus transactionnels pouvant jouer le rôle de médiateur (parmi lesquels le stress perçu) et les issues de santé (bien-être, qualité de vie, santé physique). Nous avons été confortés dans notre choix par le fait que ce modèle a été mis à l'épreuve de façon convaincante auprès de diverses populations, et notamment auprès d'étudiants plus jeunes (Boujut, 2007 ; Grebot \& Barumandzadeh, 2005).

L'intérêt de la présente étude réside dans le fait qu'elle se focalise tout particulièrement sur les antécédents du stress et leurs relations avec la santé physique chez les doctorants de diverses disciplines. Il s'agit de vérifier l'influence sur ces relations de divers facteurs tels que le sexe, la filière, la satisfaction par rapport à l'encadrement de la thèse, la pratique sportive ou la qualité du sommeil.

Compte tenu des résultats des études présentées dans notre partie théorique et de la problématique posée, nous formulons les hypothèses suivantes :

$\mathbf{H}_{1}$. Nous nous attendons à observer des scores de stress perçu plus élevés chez les doctorantes que chez leurs homologues masculins.

$\mathbf{H}_{2}$. Les doctorants qui ont une activité physique régulière obtiennent des scores de stress perçu inférieurs à ceux qui ont peu ou pas d'activité physique.

$\mathbf{H}_{3}$. Compte tenu du fait que la pratique sportive a un effet indirect sur les symptômes somatiques et que cet effet est partiellement médiatisé par le stress perçu, nous nous attendons à ce qu'une faible pratique sportive soit liée à des niveaux de stress plus élevés qui, à leur tour, prédisent davantage de symptômes somatiques.

\section{Matériel et méthodes}

\subsection{Participants et procédure}

L'étude a été réalisée à partir d'un questionnaire en ligne. Initialement, le seul critère d'inclusion était d'être inscrit en doctorat dans une université francophone. Sur 2097 réponses reçues, ont été exclues celles de doctorants d'universités autres que françaises, en nombre insuffisant pour être représentatif $(n=24)$ et 60 formulaires comportant des réponses incomplètes ou fantaisistes. Dans un second temps, nous avons également choisi d'exclure les participants âgés de plus de 40 ans afin d'éviter un biais lié à un écart d'âge trop important. L'échantillon final comprend 1923 participants (dont 67,2 \% de femmes). Leur âge moyen en juillet 2012 était de 28,04 ans 
Tableau 1

Caractéristiques de l'échantillon, moyennes et écarts-types des échelles.

\begin{tabular}{|c|c|c|c|}
\hline & Total $(n=1923)$ & $\mathrm{H}(n=630)$ & $\mathrm{F}(n=1293)$ \\
\hline & $\mathrm{M}(\sigma)$ ou pourcentage & M $(\sigma)$ ou pourcentage & $\mathrm{M}(\sigma)$ ou pourcentage \\
\hline Sexe & & $33 \%$ & $67 \%$ \\
\hline$\hat{A} g e$ & $28,04(3,18)$ & $27,84(3,09)$ & $28,15(3,22)$ \\
\hline Filière disciplinaire & & & \\
\hline Arts et lettres & 17,7 & 14 & 19,5 \\
\hline Autres sciences sociales & 20 & 14,9 & 22,4 \\
\hline Droit, économie, gestion & 3,8 & 4,1 & 3,7 \\
\hline Histoire & 10 & 9,2 & 10,4 \\
\hline Psycho, sc. de l'éduc., STAPS & 4,7 & 3,2 & 5,5 \\
\hline Santé & 21,3 & 20,3 & 21,8 \\
\hline Sciences & 22,5 & 34,3 & 16,7 \\
\hline Inscription en thèse & & & \\
\hline $1^{\mathrm{e}}$ année & 29 & 30 & 28,5 \\
\hline $2^{\mathrm{e}}$ année & 24,9 & 25,4 & 24,7 \\
\hline $3^{\mathrm{e}}$ année & 22,9 & 26,2 & 21,3 \\
\hline $4^{\mathrm{e}}$ ou $5^{\mathrm{e}}$ année & 16,8 & 13,8 & 18,3 \\
\hline $6^{\mathrm{e}}$ année ou plus & 6,3 & 4,6 & 7,2 \\
\hline Satisfaction par rapport à l'encad & & & \\
\hline Pas ou peu satisfait & 23,8 & 24,8 & 21,6 \\
\hline Moyennement satisfait. & 10,2 & 10,1 & 10,5 \\
\hline Satisfait ou très satisfait & 47,8 & 46,2 & 51,1 \\
\hline Sans réponse & 18,1 & 18,8 & 16,8 \\
\hline Qualité du sommeil & & & \\
\hline Très mauvaise & $7 \%$ & $5 \%$ & $9 \%$ \\
\hline Mauvaise & $21 \%$ & $20 \%$ & $20 \%$ \\
\hline Moyenne & $39 \%$ & $38 \%$ & $40 \%$ \\
\hline Bonne & $27 \%$ & $31 \%$ & $25 \%$ \\
\hline Très bonne & $6 \%$ & $6 \%$ & $6 \%$ \\
\hline Activité physique & & & \\
\hline Aucune & $33 \%$ & $33 \%$ & $33 \%$ \\
\hline$\approx 1$ à $3 \mathrm{~h}$ par semaine & $66 \%$ & $50 \%$ & $58 \%$ \\
\hline$>3 \mathrm{~h}$ par semaine & $11 \%$ & $17 \%$ & $9 \%$ \\
\hline Stress perçu $(P S S-14)$ & $27,51(8,76)$ & $25,6(8,67)$ & $28,43(8,65)$ \\
\hline Symptômes somatiques (SCL-90) & $20,48(5,88)$ & $18,49(5,15)$ & $21,44(5,98)$ \\
\hline
\end{tabular}

$(\sigma=3,18)$. Les filières disciplinaires ont été regroupées comme suit (voir Tableau 1) : « sciences » (doctorants en chimie, informatique, mathématiques, physique, sciences de la terre et de l'univers et sciences de l'ingénieur), 22,5\%; « arts et lettres »(doctorants en langues et littératures, philosophie, sciences du langage et de la communication), 17,7 \%, « santé » (doctorants en biologie, médecine et pharmacie), 21,3\%; « autres sciences humaines et sociales » (doctorants en anthropologie, économie, ethnologie, géographie, sciences politiques et sociologie), $20 \%$; « psycho-educ » (doctorants en psychologie, sciences cognitives et neurosciences, sciences de l'éducation et STAPS), 4,7\% et «droit, économie et gestion », 3,8\%. Plus de deux tiers des participants bénéficient d'un financement institutionnel pour effectuer leur thèse $(67,41 \%)$.

L'ensemble des données a été recueilli en ligne en juin et juillet 2012. L'information relative à cette enquête avait été diffusée auprès des responsables d'écoles doctorales recensées sur le site du ministère de l'enseignement supérieur et de la recherche 
(http://appliweb.dgri.education.fr/annuaire/selectEd.jsp), en leur demandant de bien vouloir transmettre l'information aux intéressés. Le réseau des doctorants de l'École des hautes études en sciences sociales (EHESS) a également contribué à la transmission de l'information sur des réseaux institutionnels et sociaux (listes de diffusions, Facebook, Twitter, etc.). La participation à cette enquête était basée sur le volontariat. Les données ont été exportées dans un document de tableur et recodées sous un format numérique. La recherche était présentée comme une enquête sur la qualité de vie des jeunes chercheurs. L'anonymat et la confidentialité des réponses étaient garantis.

\subsection{Instruments de mesure}

\subsubsection{Le stress perçu}

En réponse à la consigne « Diverses questions vont vous être posées ci-après. Elles concernent vos sensations et pensées au cours du mois qui vient de s'écouler. Pour chaque énoncé, indiquez à quelle fréquence il vous arrive de vous sentir ou de penser ainsi. », les participants ont complété la version française de l'échelle de stress perçu (Perceived Stress Scale, Cohen, Kamarck, \& Mermelstein, 1983).

L'échelle est constituée de 14 items. Les participants à l'enquête devaient évaluer la fréquence de ces sensations et pensées au cours des quatre dernières semaines sur une échelle de Likert en 5 points $-1=$ jamais $; 2=$ presque jamais ; $3=$ parfois $; 4=$ assez souvent $; 5=$ souvent. Un score global de stress perçu est calculé en faisant la somme des scores à chacun des 14 items. Les résultats obtenus peuvent s'échelonner théoriquement de 14 à 70.

Les qualités psychométriques de la Perceived Stress Scale en 14 items sont avérées (Cohen \& Williamson, 1988). Le coefficient d'homogénéité interne témoigne ici d'une bonne fidélité de l'échelle (alpha $=0,88)$.

\subsubsection{Les symptômes somatiques}

La sous-échelle de symptômes somatiques de la SCL-90-R (Symptom Check List 90 Revised, Somatization ; Derogatis, 1994) a été utilisée afin d'évaluer la présence de symptômes physiques chez les participants. Cette liste est constituée de 12 items, décrivant des symptômes physiques communs tels que maux de tête, vertiges, douleurs cardiaques, musculaires, difficultés respiratoires, dont il faut évaluer la gêne occasionnée pendant une période déterminée (dans notre étude, la consigne était « au cours des mois précédents »). La mesure s'effectue au moyen d'une échelle de Likert en quatre points : $1=$ pas du tout ; $2=$ un peu ; $3=$ assez ; $4=$ beaucoup. Le score est obtenu en faisant la somme des réponses à chaque item et s'échelonne théoriquement de 12 (absence de symptômes somatiques) à 48 (score maximal de symptômes somatiques). Le coefficient d'homogénéité interne témoigne ici d'une bonne fidélité de l'échelle (alpha=0,80).

\subsubsection{L'activité physique}

Concernant l'activité physique et le sommeil, dans le but de ne pas surcharger le questionnaire, et en suivant les indications figurant dans l'article de Lantian, Muller, Nurra, et Douglas (2016) sur l'utilisation d'items uniques pour mesurer certaines dimensions, nous avons fait le choix de proposer un item unique pour ces dimensions. L'activité physique a été ainsi été mesurée par la question : «Pratiquez-vous une/des activité(s) sportive(s) ? » avec un choix entre 3 réponses : 1. Non - 2. En moyenne une à trois heures par semaine -3. Plus de trois heures par semaine. 
Tableau 2

Corrélations entre les dimensions de l'âge, l'année d'inscription en thèse, l'activité physique, le sommeil, le stress perçu et les symptômes somatiques.

\begin{tabular}{|c|c|c|c|c|c|c|}
\hline & 1 & 2 & 3 & 4 & 5 & 6 \\
\hline 1. Âge & 1 & $.508^{\mathrm{b}}$ & $-.081^{\mathrm{b}}$ & $-.055^{\mathrm{a}}$ & $.089^{\mathrm{b}}$ & $.071^{\mathrm{b}}$ \\
\hline 2. Année de thèse & & 1 & -.031 & $-.063^{\mathrm{b}}$ & $.184^{\mathrm{b}}$ & $.117^{\mathrm{b}}$ \\
\hline 3. Activité physique & & & 1 & $.087^{\mathrm{b}}$ & $-.145^{\mathrm{b}}$ & $-.113^{b}$ \\
\hline 4. Sommeil & & & & 1 & $-.406^{\mathrm{b}}$ & $-.386^{\mathrm{b}}$ \\
\hline 5. Stress perçu & & & & & 1 & $.471^{\mathrm{b}}$ \\
\hline 6. Symptômes somatiques & & & & & & 1 \\
\hline
\end{tabular}

${ }^{\text {a }}$ La corrélation est significative au niveau 0,05 .

b La corrélation est significative au niveau 0,01 .

\subsubsection{La perception de la qualité du sommeil}

La qualité du sommeil a fait l'objet d'une auto-évaluation subjective au moyen de la question suivante : «Comment évaluez-vous la qualité de votre sommeil au cours du dernier mois ? » avec 5 réponses possibles : 1 . très mauvaise -2 . mauvaise -3 . moyenne -4 . bonne -5 . très bonne .

\subsubsection{Le degré de satisfaction par rapport à l'encadrement}

La satisfaction par rapport à l'encadrement a été évaluée par une question ouverte : « La direction de recherches dont vous bénéficiez correspond-elle à vos attentes ?». Les réponses ont été codées en fonction de leur contenu par deux codeurs indépendants selon trois modalités : 1. pas satisfait(e) -2 . moyennement satisfait(e) -3 . très satisfait(e). Les résultats témoignent d'une excellente fidélité inter-juges $(\mathrm{CCI}=0,98$, avec un intervalle de confiance de 0,97 à 0,99$)$. Toutefois, cette question faisait partie d'une section du questionnaire comprenant plusieurs questions ouvertes, pour lesquelles les réponses étaient facultatives, et il est à noter que 349 participants, soit $18 \%$, n’ont pas répondu à cette question.

\section{Résultats}

\subsection{Analyses préliminaires}

Nous avons utilisé Mplus 7 et SPSS 20 pour analyser les données. Dans un premier temps, nous avons procédé à différentes analyses descriptives (distribution des différentes variables). Les caractéristiques de l'échantillon sont présentées dans le Tableau 1. Afin de vérifier nos hypothèses, nous avons effectué tout d'abord une série de corrélations simples pour contrôler les interrelations entre les variables de l'étude et nous assurer que les conditions nécessaires à la vérification d'un effet médiateur étaient réunies (Tableau 2). Ensuite, une analyse de régression séquentielle a été réalisée afin de mesurer l'effet isolé et combiné des différentes variables démographiques et du stress perçu sur la santé physique, ainsi que les effets modérateurs. Finalement, afin de déterminer si l'effet des variables démographiques sur les symptômes somatiques était médiatisé par le stress perçu et par le sommeil, nous avons développé un modèle d'équations structurelles.

\subsection{Structure de l'échelle}

Nous avons effectué une analyse factorielle confirmatoire pour contrôler le modèle de mesure de la Perceived Stress Scale. Un modèle à un facteur $\left(\chi^{2}=1199,48, \mathrm{df}=77, p<0,001\right.$; 
$\mathrm{CFI}=0,868 ; \mathrm{RMSEA}=0,087$ ) présente un mauvais ajustement aux données. Un modèle à deux facteurs corrélés (correspondant aux items inversés et aux items non inversés) s'est révélé plus efficace pour expliquer les données $\left(\chi^{2}=667,10, \mathrm{df}=76, p<0,001 ; \mathrm{CFI}=0,930 ; \mathrm{RMSEA}=0,064\right)$. Cependant, le modèle qui présente le meilleur ajustement aux données est un modèle restrictif bifactoriel (Reise, Moore, \& Haviland, 2010) avec un facteur unique de stress perçu et deux facteurs orthogonaux non corrélés, reflétant la direction des items. Ce modèle confirme une dimension unique de stress perçu $\left(\chi^{2}=359,48, \mathrm{df}=63, p<0,001 ; \mathrm{CFI}=0,965 ; \mathrm{RMSEA}=0,049\right)$.

Concernant la Symptom Checklist, l'ajustement aux données d'un modèle à un seul facteur n'était pas satisfaisant $\left(\chi^{2}=529,19, \mathrm{df}=54, p<0,001 ; \mathrm{CFI}=0,856\right.$; RMSEA =0,068). Nous avons ensuite divisé l'échantillon en deux, de façon aléatoire, pour trouver des paramètres additionnels dans une partie de l'échantillon et effectué une validation croisée en utilisant l'autre partie. En nous fondant sur les indices de modification ainsi que sur l'analyse du contenu des items, nous avons introduit des covariances d'erreur pour les quatre paires d'items qui reflètent des clusters de symptômes associés à des problèmes spécifiques (items 5 et 6 pour les douleurs musculaires ; 11 et 12 pour la fatigue ; 3 et 7 pour les troubles cardiovasculaires ; 8 et 10 pour les symptômes de rhume). Il en résulte un modèle qui s'ajuste bien aux données $\left(\chi^{2}=250,15\right.$, $\mathrm{df}=50, p<0,001 ; \mathrm{CFI}=0,939 ; \mathrm{RMSEA}=0,046)$.

Les modèles résultant de ces analyses ont ensuite été utilisés pour l'analyse des variables latentes.

\subsection{Prédicteurs démographiques et comportementaux du stress perçu et des symptômes somatiques}

Afin d'évaluer les facteurs qui contribuent au stress perçu et aux symptômes somatiques, nous avons effectué des analyses de régression multiple hiérarchique avec SPSS 20. Ces analyses visaient à investiguer les prédicteurs du stress perçu, ainsi que les effets de ce dernier sur les symptômes somatiques en contrôlant les facteurs démographiques (sexe, âge), les facteurs institutionnels (année d'inscription en thèse, discipline, satisfaction par rapport à l'encadrement) et les variables liées au style de vie (activité physique, sommeil). Nous souhaitions également vérifier si les effets du stress perçu sur les symptômes somatiques étaient modérés par d'autres variables. Les résultats indiquent que les variables suivantes n'ont pas d'effet significatif sur le stress perçu : profession des parents, statut marital, présence ou non d'enfants, pays d'origine, proximité géographique de la famille. En conséquence, nous avons exclu ces variables des analyses suivantes pour simplifier le modèle.

Pour les variables nominales, un codage muet (dummy coding) a été utilisé. Les variables de prédiction du stress perçu et des symptômes somatiques ont été introduites de façon séquentielle : (1) sexe, âge ; (2) année d'inscription en thèse ; (3) filière disciplinaire (avec « sciences » comme modalité de référence) ; (4) satisfaction par rapport à l'encadrement (avec « satisfait » comme groupe de référence); (5) activité physique (avec «pas de sport» comme groupe de référence); (6) qualité du sommeil. Pour les symptômes somatiques, nous avons ajouté deux blocs de variables : (7) stress perçu ; (8) interactions du stress perçu avec les variables démographiques. Les résultats sont résumés dans le Tableau 3.

Le sexe, l'âge et l'année d'inscription en thèse sont des prédicteurs significatifs à la fois du stress perçu et des symptômes somatiques des doctorants, indiquant que les doctorantes, les étudiants plus âgés, et ceux dont la thèse dure le plus longtemps (indépendamment de leur âge) sont les individus dont le niveau de stress est le plus élevé et qui présentent le plus de symptômes somatiques. Lorsqu'on contrôle ces variables, la filière disciplinaire se révèle également liée au 
Tableau 3

Résultats des analyses de régression hiérarchique multiple prédisant le stress perçu et les symptômes somatiques chez les doctorants $(n=1923)$.

\begin{tabular}{|c|c|c|}
\hline & \multicolumn{2}{|c|}{ Variables dépendantes } \\
\hline & PSS & SCL \\
\hline Modèle complet & $\mathrm{R}^{2}=.243^{* * *}$ & $\mathrm{R}^{2}=.312^{* * *}$ \\
\hline Étape 1 & $\Delta \mathrm{R}^{2}=.030^{* * * *}$ & $\Delta \mathrm{R}^{2}=.059^{* * *}$ \\
\hline Sexe & $.147^{* * *}$ & $.233^{* * *}$ \\
\hline Âge & $.083^{* * *}$ & $.061^{* *}$ \\
\hline Étape 2 & $\Delta \mathrm{R}^{2}=.027^{* * *}$ & $\Delta \mathrm{R}^{2}=.009^{* * *}$ \\
\hline Année d'inscription en thèse & $.193^{* * *}$ & $.109^{* * *}$ \\
\hline Financement & .006 & .000 \\
\hline Étape 3 : discipline & $\Delta \mathrm{R}^{2}=.009^{* *}$ & $\Delta \mathrm{R}^{2}=.007^{*}$ \\
\hline Arts et lettres & $.110^{* * * *}$ & $.102^{* * *}$ \\
\hline Histoire & .030 & .024 \\
\hline Autres SHS & $.077^{* *}$ & .052 \\
\hline Droit, économie, gestion & .040 & .025 \\
\hline Santé & $.078^{* *}$ & .050 \\
\hline Psycho, sc. de l'éduc., STAPS & $.064^{* *}$ & .005 \\
\hline Étape 4 : satisfaction/encadrement & $\Delta \mathrm{R}^{2}=.038^{* * *}$ & $\Delta \mathrm{R}^{2}=.015^{* * *}$ \\
\hline NA & .038 & .006 \\
\hline Moyennement satisfait & $.094^{* * *}$ & .038 \\
\hline Non satisfait & $.205^{* * *}$ & $.127^{* * *}$ \\
\hline Étape 5 : activité physique & $\Delta \mathrm{R}^{2}=.018^{* * *}$ & $\Delta \mathrm{R}^{2}=.010^{* * *}$ \\
\hline 1 à 3 heures hebdomadaires & $-.118^{* * *}$ & $-.050^{*}$ \\
\hline Plus de 3 heures hebdomadaires & $-.130^{* * *}$ & $-.112^{* * *}$ \\
\hline Étape 6 : sommeil & $\Delta \mathrm{R}^{2}=.127^{* * *}$ & $\Delta \mathrm{R}^{2}=.124^{* * *}$ \\
\hline Qualité perçue du sommeil & $-.365^{* * *}$ & $-.360^{* * *}$ \\
\hline Étape $7:$ stress perçu (PSS) & - & $\Delta \mathrm{R}^{2}=.083^{* * *}$ \\
\hline Stress perçu & & $.332^{* * *}$ \\
\hline Étape $8: P S S \times v$. socio-démo. & - & $\Delta \mathrm{R}^{2}=.013^{* * *}$ \\
\hline PSS $\times$ sexe & & $.086^{* *}$ \\
\hline PSS $\times$ discipline & & $-.066^{* * *}$ \\
\hline PSS $\times$ qualité perçue du sommeil & & $-.078^{* * *}$ \\
\hline
\end{tabular}

**** $p<0,001 ;{ }^{* *} p<0,01 ;{ }^{*} p<0,05$. Les coefficients de régression standardisés pour les variables introduites à chaque étape sont indiqués.

stress, mais sa relation aux symptômes est moins prégnante. Comparé au groupe de référence («sciences »), dont le niveau de stress est le moins élevé, les filières où les doctorants présentent des niveaux de stress plus élevés sont : " arts et lettres », " santé » " autres sciences humaines et sociales » et «psychologie, sciences de l'éducation, STAPS ». Le niveau de symptômes somatiques est significativement plus élevé uniquement dans le premier groupe. Le degré de satisfaction par rapport à l'encadrement est également un facteur prédictif significatif du stress : les doctorants peu ou moyennement satisfaits de l'encadrement dont ils bénéficient présentent un niveau de stress plus élevé que ceux qui sont satisfaits. En outre, l'insatisfaction est également liée à un niveau plus élevé de symptômes somatiques, ce qui semble indiquer que des difficultés majeures avec le directeur de thèse sont également susceptibles d'affecter la santé des doctorants. L'activité physique, même limitée, est négativement liée au stress. Toutefois, seule une pratique supérieure à trois heures par semaine permet de réduire significativement les symptômes somatiques. Enfin, la qualité perçue du sommeil est un prédicteur significatif du stress perçu aussi bien que de 
la santé physique. Par conséquent, sacrifier son sommeil durant la thèse peut avoir des effets particulièrement délétères.

\subsection{Les effets du stress perçu sur les symptômes somatiques et ses modérateurs}

Nous nous sommes également intéressés aux effets du stress perçu sur les symptômes somatiques et aux variables démographiques qui pouvaient le modérer, indiquant des groupes spécifiques potentiellement plus à risque que les autres. En contrôlant les variables introduites dans les étapes 1 à 6 , le stress perçu s'est révélé un assez bon prédicteur des symptômes somatiques. L'on pourrait objecter qu'un mauvais sommeil peut constituer en soi un symptôme de stress perçu ; c'est pourquoi nous avons répété l'analyse combinant les étapes 6 et $7\left(\Delta \mathrm{R}^{2}=.207\right.$, $p<0,001)$. Les résultats indiquent que le stress perçu $(\beta=.332, p<0,001)$ aussi bien que la qualité du sommeil $(\beta=-.239, p<0,001)$ prédisent une variance unique dans les symptômes. La contribution plus faible du sommeil, mais qui demeure significative, indique que seule une partie de sa variance partagée avec les symptômes somatiques est expliquée par le stress perçu.

Nous avons aussi exploré les effets d'interaction entre le stress perçu et l'ensemble des variables introduites dans les étapes 1 à 6 . La proportion incrémentale de la variance expliquée par l'ensemble des 16 termes en interaction est assez faible, mais statistiquement significative $\left(\Delta \mathrm{R}^{2}=.016, p \leq .001\right)$. Seules trois des variables socio-démographiques interagissent de façon significative avec le stress perçu pour expliquer le niveau de symptômes somatiques et ont été retenues. Le sexe - les doctorantes sont plus susceptibles d'éprouver les symptômes somatiques du stress que les doctorants -, la filière disciplinaire «psychologie, sciences de l'éducation, STAPS », ce qui suggère que les doctorants de cette filière manifestent moins de symptômes somatiques liés au stress que les autres, et enfin, l'interaction avec la qualité perçue du sommeil, indiquant que, outre leurs effets spécifiques, la combinaison d'un mauvais sommeil et un niveau de stress élevé peut avoir un effet additionnel sur la santé somatique.

\subsection{Le rôle médiateur du stress perçu}

Nous avons ensuite vérifié si le stress perçu exerçait un effet médiateur entre les variables démographiques et la santé. À cette fin, nous avons modélisé l'échelle de stress perçu et la liste de symptômes comme variables latentes basées sur les modèles de mesure décrits ci-dessus. Le modèle combiné pour les doctorants regroupés en cluster disciplinaire, en utilisant une approche par modélisation d'équation structurelle sous Mplus 7,2 (utilisant l'option « type = complex » pour des échantillons groupés) présente un bon ajustement aux données $\left(\chi^{2}=983,56, \mathrm{df}=280\right.$; $\mathrm{CFI}=.953 ; \mathrm{RMSEA}=.036$ ). Nous avons émis l'hypothèse que la mauvaise qualité du sommeil pouvait être une conséquence du stress perçu aussi bien qu'un mécanisme indépendant reliant la charge de travail aux problèmes de santé des doctorants. Dans ce modèle théorique, l'ajustement aux données démographiques était légèrement moins bon $\left(\chi^{2}=1487,25\right.$, df $=429 ; \mathrm{CFI}=.938$; RMSEA =.036). En nous fondant sur l'analyse des indices de modification, nous avons ajouté un effet direct du sexe sur les symptômes $\left(\chi^{2}=59,07 ; p<0,001\right)$, en conformité avec les résultats d'autres études où une différence entre hommes et femmes est observée sur les scores de la SCL90 (Urbán et al., 2014). Il en résulte un modèle mieux ajusté aux données ( $\chi^{2}=1427,48$, df $=428$; $\mathrm{CFI}=.941 ; \mathrm{RMSEA}=.035)$, qui ne met en lumière aucun effet significatif supplémentaire associé aux effets directs des variables démographiques sur les symptômes somatiques.

Les paramètres standardisés de ce modèle sont représentés sur la Fig. 1 (seules les trajectoires statistiquement significatives à $p<0,05$ sont indiquées), afin de montrer les effets des prédicteurs 


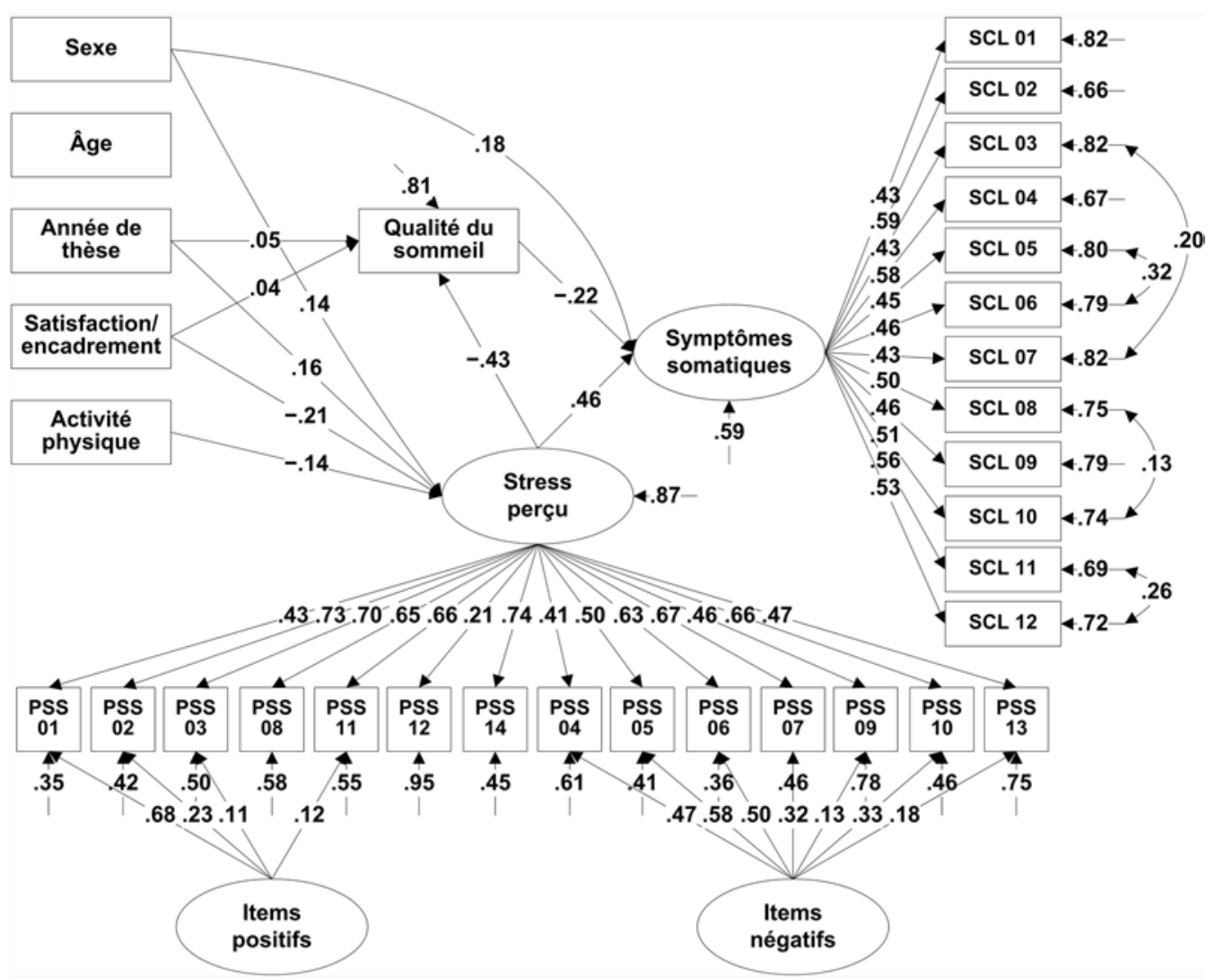

Fig. 1. Modèle de médiation partielle résultant de nos analyses (les voies standardisées sont indiquées).

observés sur le facteur latent de symptômes somatiques, qui sont partiellement médiatisés par la qualité du sommeil et le facteur latent de stress perçu.

Le modèle indique que l'effet du stress perçu sur les symptômes somatiques est partiellement médiatisé par la qualité du sommeil, qui diminue lorsque le stress augmente. En dehors de l'effet du sexe, tous les effets des autres variables socio-démographiques et comportementales sont médiatisés par le stress perçu et la qualité du sommeil. Bien que l'effet des variables sociodémographiques sur la qualité du sommeil soit principalement expliqué par le stress, la satisfaction par rapport à l'encadrement et l'année d'inscription en thèse ont des effets significatifs spécifiques sur la qualité du sommeil, ce qui indique que la qualité du sommeil serait moins bonne lorsqu'ils sont peu satisfaits de l'encadrement dont ils bénéficient, mais elle est meilleure chez les doctorants les plus avancés dans la thèse.

Nous avons poursuivi les analyses en testant les effets indirects (les valeurs standardisées STDYX sont indiquées). L'effet indirect du sexe sur les symptômes somatiques $(\beta=.074$, $p<0,001)$ était entièrement médiatisés par le stress perçu $(\beta=.065, p<0,001)$, mais non par la qualité du sommeil. Les effets indirects du sexe sont, dans ce modèle et dans cette population, expliqués par le fait que la perception de situations stressantes diffère considérablement entre hommes et femmes, et cette évaluation subjective du stress agit à son tour de façon significative sur les symptômes. La qualité du sommeil en revanche ne se différencie pas suffisamment entre 
hommes et femmes pour que l'effet que celle-ci peut avoir sur les symptômes en soit affecté. Un effet similaire a été observé pour l'effet indirect du sport sur les symptômes somatiques $(\beta=-.085, p<0,001)$, qui était entièrement médiatisé par le stress perçu $(\beta=-.065, p<0,001)$. Cependant, les effets indirects de la satisfaction par rapport à l'encadrement $(\beta=-.125, p<0,001)$ et l'année d'inscription en thèse $(\beta=.081, p<0,01)$ étaient seulement partiellement médiatisés par le stress perçu ( $\beta=-.096, p<0,001$ et $\beta=.075, p<0,001$, respectivement), témoignant d'un effet de médiation par la qualité du sommeil faible, mais significatif. Ces résultats indiquent que, bien que ce soit principalement le stress qui explique la mauvaise qualité de sommeil chez les doctorants, dans certains cas, la qualité du sommeil est affectée par l'approche de l'échéance de la thèse ou par l'insatisfaction par rapport à l'encadrement, qui ont à leur tour des effets - faibles mais significatifs - sur la santé somatique.

\section{Discussion}

L'objectif principal de cette recherche était d'explorer les relations entre stress perçu et symptômes somatiques dans un échantillon de doctorants inscrits dans des universités françaises, tout en vérifiant l'influence sur ces interrelations de facteurs tels que le genre des personnes, la filière disciplinaire, la pratique sportive et la perception de la qualité du sommeil. Le premier constat est que la plus grande vulnérabilité au stress perçu des doctorantes par rapport à leurs homologues masculins est confirmée : il y a bien un effet du genre sur la moyenne des scores obtenus aux deux questionnaires. La corrélation positive entre un niveau élevé de stress perçu et la présence de symptômes somatiques se vérifie également, ce qui corrobore les liens observés par d'autres études entre le niveau de stress et les plaintes somatiques (Dahlgren, Kecklund, Theorell, \& Åkerstedt, 2009 ; Lovell et al., 2011) ou la résistance aux infections (Cohen, Doyle, \& Skoner, 1999).

Le rôle médiateur du stress perçu entre l'activité physique et les symptômes somatiques est présent, même si l'effet est relativement faible. Rappelons que les participants sont plutôt jeunes (moins de 40 ans) et que les effets délétères de l'absence d'activité physique sur la santé, tout comme ceux du stress, s'accentuent avec l'âge. Les participants qui évaluent la qualité de leur sommeil comme satisfaisante obtiennent des scores de stress perçu moins élevés que les autres.

L'auto-évaluation de la qualité du sommeil, de l'activité physique et de la satisfaction par rapport à l'encadrement repose ici sur un seul item. Dans le cas de l'encadrement, en particulier, les réponses reflètent peut-être davantage les attentes des intéressés que leur satisfaction par rapport à l'encadrement dont ils bénéficient. Toutefois, la fiabilité de l'évaluation à l'aide d'un item unique est reconnue dans de nombreux cas, comme par exemple pour l'estime de soi (Robins, Hendin, \& Trzesniewski, 2001) et l'anxiété (Davey, Barratt, Butow, \& Deeks, 2007). En outre, dans le cas d'une étude qui se veut exploratoire, le gain en termes de facilité d'administration du questionnaire nous a semblé suffisant pour compenser les inconvénients potentiels liés au risque d'erreur commise par les participants dans leur réponse (Rammstedt \& Beierlein, 2014).

Les résultats sont cohérents avec d'autres recherches menées en population étudiante et auprès de jeunes adultes : il existe un lien étroit entre le stress et la qualité du sommeil (ex., Brand et al., 2010 ; Lund et al., 2010). Les personnes concernées par les problèmes de sommeil ne sont pas toujours consciente de leurs difficultés, d'où l'importance d'une large diffusion de l'information sur le sommeil et, plus généralement, sur ses relations avec l'hygiène de vie dans les universités (Lund et al., 2010 ; Pallos et al., 2007).

Le seul effet qui n'ait pas été prévu théoriquement est l'association positive de la qualité du sommeil avec l'année d'inscription en thèse. Dans la mesure où l'effet est faible $(\beta=.05)$, ce 
résultat peut constituer un simple artefact statistique des données ou refléter la réalité (dormir davantage peut constituer une stratégie de coping face à un stress accru). Les études futures gagneront à utiliser des mesures de qualité du sommeil plus précises et prenant en compte sa durée, de façon à évaluer si le sommeil des doctorants évolue différemment en fin de thèse et si cela affecte leur santé.

Quant à l'effet indirect de l'activité physique sur les symptômes somatiques, il passe également par le stress perçu. Les résultats de la présente étude indiquent que, même si une pratique minimale d'activité physique contribue à réduire le stress perçu, seule une pratique plus soutenue permet de réduire les effets du stress sur la santé physique. Il serait intéressant de prendre également en compte l'activité physique non sportive, telle que les déplacements non motorisés, qui contribuent à une meilleure santé physique et mentale. Il semble cependant que l'influence de médiateurs soit rarement recherchée en dehors des travaux qui s'inscrivent explicitement dans le cadre théorique de la psychologie de la santé et de ce point de vue, la présente recherche apporte des éléments de compréhension significatifs au sujet d'une population peu étudiée (Boujut \& Décamps, 2012).

Ce travail souffre néanmoins de plusieurs limitations liées en particulier au mode de recrutement des participants, au moment de l'enquête, à la méthode d'enquête qui repose entièrement sur des auto-évaluations, ainsi qu'à son caractère transversal. Les questionnaires ont été mis en ligne et l'annonce a été largement diffusée, notamment grâce à l'implication des écoles doctorales et d'un certain nombre de doctorants. Il est donc impossible d'estimer le taux de réponses par rapport au nombre de personnes qui ont reçu l'information. Il est tout aussi difficile de connaître avec précision les motivations des participants qui ont pris le temps de répondre à l'étude et de savoir, par conséquent, si les résultats concernent un échantillon représentatif des doctorants français. En effet, il existe un déséquilibre entre les filières : les doctorants en psychologie ou sciences de l'éducation, par exemple, ne sont pas très représentés (peut-être les doctorants en psychologie et en sciences de l'éducation sont-ils davantage sollicités que d'autres au cours de leurs études, ce qui pourrait expliquer leur manque d'enthousiasme à l'idée de participer à une enquête supplémentaire). Notre échantillon comprend des doctorants à tous les stades de la thèse, certains venant de s'inscrire, d'autres ayant dépassé sept ans (généralement inscrits en lettres ou en sciences humaines et exerçant une activité professionnelle parallèlement à leur recherche doctorale). Il aurait été intéressant de contrôler ce biais.

L'enquête a eu lieu au tout début de l'été, en période de congé scolaire (entre le 29 juin et le 14 juillet), ce qui a pu influencer les résultats. Toutefois, le doctorant n'est pas dans une situation comparable à celle d'un étudiant de premier cycle : il n'est pas soumis au même type de calendrier ni aux mêmes évaluations que ce dernier et il met bien souvent à profit la période estivale, où il est libéré de certaines contraintes (enseignement, séminaires) pour travailler intensivement sur sa thèse. En outre, les questionnaires qui évaluaient des dimensions limitées dans le temps portaient sur le dernier mois, c'est-à-dire qu'ils englobaient le mois de juin, où les activités universitaires sont généralement plutôt denses.

\section{Conclusion}

Cette étude sur les doctorants apporte un certain nombre d'éléments significatifs permettant de mieux cerner leur expérience spécifique. Elle montre notamment que les doctorants n'échappent pas au «mal-être étudiant » et donne un aperçu de l'ampleur des recherches qui restent à mener. En effet, nombreuses sont les dimensions qui ont été écartées dans ce travail exploratoire. Il sera nécessaire de le compléter en investiguant d'autres facteurs comme les tracas quotidiens, les addictions, les comportements alimentaires, les stratégies d'ajustement (coping), la régulation 
émotionnelle, la tendance à la procrastination et la dépression et le soutien social. Il est établi qu'ils ont un impact direct sur le stress et sur la santé des étudiants (Clark, Murdock, \& Koetting, 2009 ; Delelis, Christophe, Berjot, \& Desombre, 2011 ; Martin \& Dahlen, 2005 ; Osiurak et al., 2015 ; Torres \& Solberg, 2001 ; Wohlgemuth \& Betz, 1991) mais leurs effets sur celle des doctorants n'ont pas fait l'objet de recherches. Il sera également nécessaire d'approfondir l'étude des liens entre activité physique et stress, l'activité physique ne correspondant pas nécessairement à une activité sportive. Par ailleurs, dans notre étude les conditions financières n'avaient pas d'impact sur le stress perçu ou les symptômes physiques, mais cette question devraient être investiguée plus en détail afin d'identifier au mieux les facteurs de risque et de protection des doctorants. D'autres prolongements possibles de cette recherche pourraient consister, d'une part, à suivre une cohorte de doctorants de l'inscription à la fin de leur doctorat dans le cadre d'une étude longitudinale, de l'autre, de mener une enquête permettant de comparer ces données avec celles concernant de jeunes adultes du même âge en dehors du milieu universitaire.

Le présent article montre aussi qu'il est urgent de mener des interventions concrètes auprès de cette population : le besoin d'informations des étudiants en général à propos des bienfaits des activités physiques est fréquemment souligné (Boujut \& Décamps, 2012 ; Katz, Davis, \& Findlay, 2002) et les conclusions auxquelles aboutissent les chercheurs prennent de façon unanime la forme de plaidoyers en faveur de programmes de psycho-éducation visant à promouvoir la qualité de vie, le bien-être, ainsi que la santé physique et mentale, qu'il s'agisse des étudiants (Boujut \& Décamps, 2012 ; Mazé \& Verlhiac, 2013 ; Neveu et al., 2012; Parks, 2013) ou des doctorants (Armstrong, 2014 ; Hill, 2011 ; El-Ghoroury et al., 2012). C'est d'autant plus nécessaire que les étudiants sont effectivement réceptifs aux démarches psycho-éducatives (Frank, Galuska, Elon, \& Wright, 2004 ; Melo-Carrillo, Van Oudenhove, \& Lopez-Avila, 2012).

L'élaboration de programmes d'intervention pourra s'inspirer de travaux sur la « manière de prendre soin de soi » (self-care practices, cf. Myers et al., 2012), c'est-à-dire l'ensemble des comportements qui préservent et favorisent le bien-être physique et émotionnel, qui peuvent inclure la qualité du sommeil, l'activité physique, le recours au soutien social, de stratégies de régulations émotionnelles ou la pratique de la pleine conscience (Godfrey et al., 2011). Une attention particulière devrait être accordée à la relation d'encadrement, qui joue un rôle important par rapport à la persistance dans le cursus (Devos et al., 2016 ; Litalien \& Guay, 2015). Plus largement, la psychologie positive, en raison de l'importance qu' elle accorde à l'évaluation scientifique des résultats d'intervention, offre un cadre rigoureux permettant de structurer les programmes en s'inspirant d'expérimentations réussies dans d'autres domaines (Martin-Krumm, Tarquinio, \& Shaar, 2013 ; Peillod-Book \& Shankland, 2016). Différentes interventions en psychologie positive auprès de doctorants sont d'ailleurs en cours (Haag, 2015 ; Marais, Flault, Juniper, Haag, \& Shankland, 2016). Il serait donc intéressant de recenser les principales initiatives actuelles dans l'enseignement supérieur pour en évaluer l'efficacité, notamment en ayant recours à des méthodes mixtes (voir Bosisio \& Santiago-Delefosse, 2014). Le moment le plus propice à la sensibilisation serait assurément le début de la thèse, voire la fin du master. En effet, les résultats de notre étude indiquent qu'à l'approche de la fin du cursus doctoral, les problèmes de santé physique et le stress augmentent. C'est donc en amont de cette phase qu'il convient d'agir sur les comportements de santé des doctorants par le biais de techniques de régulation du stress et des comportements de santé ou encore en agissant sur un plan plus systémique auprès des directeurs de thèse en vue d'une amélioration de la satisfaction de l'encadrement et de la réduction du stress lié à celui-ci. Dans cette perspective, il sera nécessaire de prendre en considération la situation spécifique des doctorants, qui sont confrontés à un monde de l'emploi en pleine mutation et à des difficultés socio-économiques croissantes. Ceux-ci se trouvent donc particulièrement exposés aux risques 
psychosociaux et la plus grande attention doit être accordée à l'accompagnement en vue de leur insertion professionnelle.

\section{Déclaration de liens d'intérêts}

Les auteurs déclarent ne pas avoir de liens d'intérêts.

\section{Remerciements}

Les auteurs remercient tous les participants à cette enquête, ainsi que les écoles doctorales et le réseau des doctorants de l'École des hautes études en sciences sociales, qui ont contribué à la transmission de l'information sur des réseaux institutionnels et sociaux.

\section{Références}

Abdulghani, H. M., Alrowais, N. A., Bin-Saad, N. S., Al-Subaie, N. M., Haji, A. M. A., \& Alhaqwi, A. I. (2012). Sleep disorder among medical students: Relationship to their academic performance. Medical Teacher, 34(Suppl. 1), 37-41.

Aktekin, M., Karaman, T., Senol, Y. Y., Erdem, S., Erengin, H., \& Akaydin, M. (2001). Anxiety, depression and stressful life events among medical students: A prospective study in Antalya, Turkey. Medical Education, 35(1), 12-17.

Alonso, J., Angermeyer, M. C., Bernert, S., Bruffaerts, R., Brugha, T. S., Bryson, H., et al. (2004). Prevalence of mental disorders in Europe: Results from the European Study of the Epidemiology of Mental Disorders (ESEMeD) project. Acta psychiatrica Scandinavica. Supplementum, 420, 21-27.

Armstrong, J. (2014). Stress, coping, and progression through doctoral psychology programs. San Francisco Campus: Thèse de doctorat Alliant International University, California School of Professional Psychology.

Aspland, T., Edwards, H., O’Leary, J., \& Ryan, Y. (1999). Tracking new directions in the evaluation of postgraduate supervision. Innovative Higher Education, 24(2), 127-147.

Austin, A. (2002). Preparing the next generation of faculty: Graduate school as socialization to the academic career. The Journal of Higher Education, 73, 94-122.

Barnett, J. E., Baker, E. K., Elman, N. S., \& Schoener, G. R. (2007). In pursuit of wellness: The self-care imperative. Professional Psychology: Research and Practice, 38(6), 603-612.

Benesek, J. P. (1998). Stress and coping among psychology doctoral students: A comparison of self-reported stress levels and coping styles of Ph.D. and Psy.D. students. Thèse de doctorat University of Hartford.

Bhui, K. (2002). Physical activity and stress. In S. A. Stansfeld, \& M. G. Marmot (Eds.), Stress and the heart: Psychosocial pathways to coronary heart disease (pp. 158-167). Williston, VT: BMJ Books.

Bireda, A. D. (2015). Challenges to the doctoral journey: A case of female doctoral students from Ethiopia. Open Praxis, 7(4), 287-297.

Bosisio, F., \& Santiago-Delefosse, M. (2014). Intérêts et limites de l'utilisation d'une méthodologie mixte : à propos d'une recherche en psychologie de la santé. Pratiques psychologiques, 20(1), 39-53.

Bouchard, C., Blair, S. N., \& Haskell, W. L. (2012). Physical activity and health. Champaign, IL: Human Kinetics.

Boudoukha, A. H., Bonnaud-Antignac, A., Acier, D., \& Chaillou, A. (2011). Anxiété et stress : impact de la préparation du concours de pharmacie. Journal de thérapie comportementale et cognitive, 21(3), 90-96.

Boujut, E. (2007). Facteurs prédisant le développement de symptômes dépressifs, de symptômes somatiques, de troubles des conduites alimentaires et de l'échec académique chez des étudiants de première année. In Une étude prospective en psychologie de la santé. Thèse de doctorat Université de Bordeaux.

Boujut, É., \& Décamps, G. (2012). Relations entre les émotions négatives, l'estime de soi, l'image du corps et la pratique sportive des étudiants de première année. Journal de thérapie comportementale et cognitive, 22(1), 16-23.

Boujut, E., Koleck, M., Bruchon-Schweitzer, M., \& Bourgeois, M.-L. (2009). La santé mentale chez les étudiants : suivi d'une cohorte en première année d'université. Annales médico-psychologiques, revue psychiatrique, 167(9), 662-668.

Brand, S., Gerber, M., Beck, J., Hatzinger, M., Pühse, U., \& Holsboer-Trachsler, E. (2010). High exercise levels are related to favorable sleep patterns and psychological functioning in adolescents: A comparison of athletes and controls. Journal of Adolescent Health, 46(2), 133-141.

Bruchon-Schweitzer, M. (2002). Psychologie de la santé modèles, concepts et méthodes. Paris: Dunod. 
Cherewatenko, V. S., \& Perry, P. (2003). The stress cure: A simple 7-step plan to balance mood, improve memory, and restore energy. New York: HarperResource.

Clark, H. K., Murdock, N. L., \& Koetting, K. (2009). Predicting burnout and career choice satisfaction in counseling psychology graduate students. Counseling Psychologist, 37(4), 580-606.

Cohen, S., Doyle, W. J., \& Skoner, D. P. (1999). Psychological stress, cytokine production, and severity of upper respiratory illness. Psychosomatic Medicine, 61(2), 175-180.

Cohen, S., Kamarck, T., \& Mermelstein, R. (1983). A global measure of perceived stress. Journal of Health \& Social Behavior, 24(4), 385-396.

Cohen, S., \& Williamson, G. (1988). Perceived stress in a probability sample of the U.S. Dans S. Spacapam et S. Oskamp (Dir.), The social psychology of health: Claremont Symposium on Applied Social Psychology (p. 31-67), Newbury Park, CA: Sage.

Dahlgren, A., Kecklund, G., Theorell, T., \& Åkerstedt, T. (2009). Day-to-day variation in saliva cortisol - relation with sleep, stress and self-rated health. Biological Psychology, 82(2), 149-155.

Davey, H. M., Barratt, A. L., Butow, P. N., \& Deeks, J. J. (2007). A one-item question with a Likert or Visual Analog Scale adequately measured current anxiety. Journal of Clinical Epidemiology, 60(4), 356-360.

Davis, M. C., Burleson, M. H., \& Kruszewski, D. M. (2011). In R. Contrada, \& A. Baum (Eds.), Gender: Its relationship to stressor exposure, cognitive appraisal/comping processes, stress responses, and health outcomes (pp. 247-261).

Delelis, G., Christophe, V., Berjot, S., \& Desombre, C. (2011). Stratégies de régulation émotionnelle et de coping : quels liens ? Bulletin de psychologie, 64(5), 471-479.

Derogatis, L. R. (1994). Administration, scoring and procedures. Manual III (3 ed). Minneapolis, MN: National Computer Systems.

Devos, C., Boudrenghien, G., Van der Linden, N., Azzi, A., Frenay, M., Galand, B., et al. (2016). Doctoral students' experiences leading to completion or attrition: A matter of sense, progress and distress. European Journal of Psychology of Education, 32, 61-77.

Dinger, M. K. (1999). Physical activity and dietary intake among college students. American Journal of Health Studies, 15(3), 139-148.

El-Ghoroury, N. H., Galper, D. I., Sawaqdeh, A., \& Bufka, L. F. (2012). Stress, coping, and barriers to wellness among psychology graduate students. Training and Education in Professional Psychology, 6(2), 122-134.

Elman, N. S., \& Forrest, L. (2007). From trainee impairment to professional competence problems: Seeking new terminology that facilitates effective action. Professional Psychology: Research and Practice, 38(5), 501-509.

Flynn, S. M., Schipper, L. J., Roach, A. R., \& Segerstrom, S. C. (2009). Gender differences in delayed-type hypersensitivity response: Effects of stress and coping in first-year law students. Brain, Behavior \& Immunity, 23(5), $672-676$.

Fogg, P. (2009). Grad-School Blues. The Chronicle of Higher Education.. http://chronicle.com/article/ Grad-School-Blues/29566

Frank, E., Galuska, D. A., Elon, L. K., \& Wright, E. H. (2004). Personal and clinical exercise-related attitudes and behaviors of freshmen U.S. medical students. Research Quarterly For Exercise And Sport, 75(2), 112-121.

Gérard, L. (2014). Le doctorat: un rite de passage. Paris: Téraèdre.

Godfrey, C. M., Harrison, M. B., Lysaght, R., Lamb, M., Graham, I. D., \& Oakley, P. (2011). Care of self - care by other care of other: The meaning of self-care from research, practice, policy and industry perspectives. International Journal Of Evidence-Based Healthcare, 9(1), 3-24.

Golde, C. (1998). Beginning graduate school: Explaining first-year doctoral attrition. New Directions for Higher Education, 101, 55-64.

Grady, R. K., La Touche, R., Oslawski-Lopez, J., Powers, A., \& Simacek, K. (2014). Betwixt and between: The social position and stress experiences of graduate students. Teaching Sociology, 42(1), 5-16.

Grebot, E., \& Barumandzadeh, T. (2005). L'accès à l'université : une situation stressante à l'origine de certaines stratégies d'ajustement dysfonctionnelles. Annales médico-psychologiques, revue psychiatrique, 163(7), 561-567.

Haag, P. (2015). Well-being, health and work during the PhD: A pilot participative action research. http://www.ipositive-education.net/well-being-health-and-work-during-the-phd-a-pilot-participative-action-research/

Hammoud, S. (2005). Étudiants: tous stressés ? : MGEL - La Mutuelle Étudiante - Prévention Santé.

Hawley, P. (1993). Being bright is not enough. The unwritten rules of doctoral study.

Helmers, K. F., Danoff, D., Steinert, Y., Young, S. N., \& Leyton, M. (1997). Stress and depressed mood in medical students, law students, and graduate students at McGill university. Academic Medicine, 72(8), 708-714.

Hill, L. M. (2011). Perceived stress, academic support, social support, and professional support factors as predictors of student success in distributed-learning doctoral education. Thèse de doctorat Fielding Graduate University. 
Johnson, B., Batia, A., \& Haun, J. (2008). Perceived stress among graduate students: Roles, responsibilities, \& social support. Virgina Journal, 29(3), 31-36.

Katz, A., Davis, P., \& Findlay, S. S. (2002). Ask and ye shall plan: A health needs assessment of a university population. Canadian Journal of Public Health, 93, 63-66.

Kline, C. E. (2014). The bidirectional relationship between exercise and sleep implications for exercise adherence and sleep improvement. American Journal of Lifestyle Medicine, 8(6), 375-379.

Knox, S., Burkard, A. W., Janecek, J., Pruitt, N. T., Fuller, S. L., \& Hill, C. E. (2011). Positive and problematic dissertation experiences: The faculty perspective. Counselling Psychology Quarterly, 24(1), 55-69.

Kurtz-Costes, B., Helmke, L. A., \& Ulku-Steiner, B. (2006). Gender and doctoral studies: The perceptions of Ph.D. students in an American University. Gender \& Education, 18(2), 137-155.

Ladner, J., Mihailescu, S., Kern, L., Romo, L., \& Tavolacci, M. (2016). Burn out in university students: Time now for implementing new public health approaches. European Journal of Public Health, 26(Suppl. 1), 161-162.

Lantian, A., Muller, D., Nurra, C., \& Douglas, K. M. (2016). Measuring belief in conspiracy theories: Validation of a French and English Single-Item Scale. International Review of Social Psychology, 29(1), 1-14.

Lazarus, R. S., \& Folkman, S. (1984). Stress, appraisal, and coping. New York: Springer publishing company.

Litalien, D., \& Guay, F. (2015). Dropout intentions in PhD studies: A comprehensive model based on interpersonal relationships and motivational resources. Contemporary Educational Psychology, 41, 218-231.

Liu, C., Xie, B., Chou, C.-P., Koprowski, C., Zhou, D., Palmer, P., et al. (2007). Perceived stress, depression and food consumption frequency in the college students of China seven cities. Physiology \& Behavior, 92(4), $748-754$

Longfield, A., Romas, J., \& Irwin, J. D. (2006). The self-worth, physical and social activities of graduate students: A qualitative study. College Student Journal, 40(2), 282-292.

Lovallo, W. R. (2005). Stress and health: Biological and psychological interactions (2nd ed.). Thousand Oaks, CA: Sage.

Lovell, B., Moss, M., \& Wetherell, M. A. (2011). Perceived stress, common health complaints and diurnal patterns of cortisol secretion in young, otherwise healthy individuals. Hormones and Behavior, 60(3), 301-305.

Lovitts, B. E., \& Nelson, C. (2000). The hidden crisis in graduate education: Attrition from Ph.D. programs. Academe, 86(6), 44-50.

Lund, H. G., Reider, B. D., Whiting, A. B., \& Prichard, J. R. (2010). Sleep patterns and predictors of disturbed sleep in a large population of college students. Journal of Adolescent Health, 46(2), 124-132.

Marais, G., Flault, R., Juniper, B., Haag, P., \& Shankland, R. (2016). Career uncertainty undermines well-being of French PhD students and what positve psychology can do about it. Poster présenté à la $8^{\mathrm{e}}$ European Conference on Positive Psychology (Angers).

Martin-Krumm, C., Tarquinio, C., \& Shaar, M.-J. (2013). Psychologie positive en environnement professionnel. Bruxelles: De Boeck.

Martin, R. C., \& Dahlen, E. R. (2005). Cognitive emotion regulation in the prediction of depression, anxiety, stress, and anger. Personality and Individual Differences, 39(7), 1249-1260.

Matos, F. (2013). PhD and the manager's dream: Professionalising the students, the degree and the supervisors? Journal of Higher Education Policy and Management, 35(6), 626-638.

Mazé, C., \& Verlhiac, J.-F. (2013). Stress et stratégies de coping d'étudiants en première année universitaire : rôles distinctifs de facteurs transactionnels et dispositionnels. Psychologie française, 58(2), 89-105.

McCleary, R., \& Zucker, E. L. (1991). Higher trait- and state-anxiety in female law students than male law students. Psychological Reports, 68(2), 1075-1078.

McKinzie, C., Altamura, V., Burgoon, E., \& Bishop, C. (2006). Exploring the effect of stress on mood, self-esteem, and daily habints with psychology graduate students. Psychological Reports, 99(2), 439-448.

Melo-Carrillo, A., Van Oudenhove, L., \& Lopez-Avila, A. (2012). Depressive symptoms among Mexican medical students: High prevalence and the effect of a group psychoeducation intervention. Journal of Affective Disorders, 136(3), 1098-1103.

Mezick, E. J., Matthews, K. A., Hall, M., Kamarck, T. W., Buysse, D. J., Owens, J. F., et al. (2009). Intraindividual variability in sleep duration and fragmentation: Associations with stress. Psychoneuroendocrinology, 34(9), 1346-1354.

Migeot, V., Ingrand, I., Defossez, G., Salardaine, F., Lahorgue, M. F., Poupin, C., et al. (2006). Comportements de santé des étudiants d'IUT de l'Université de Poitiers. Santé publique, 18(2), 195-205.

Myers, S. B., Sweeney, A. C., Popick, V., Wesley, K., Bordfeld, A., \& Fingerhut, R. (2012). Self-care practices and perceived stress levels among psychology graduate students. Training and Education in Professional Psychology, $6(1), 55-66$. 
Neveu, D., Doron, J., Visier, L., Boiché, J., Trouillet, R., Dujols, P., et al. (2012). Students perceived stress in academic programs: Consequences for its management. Revue d'épidémiologie et de santé publique, 60(4), $255-264$.

Nguyen-Michel, S., Unger, J. B., Hamilton, J., \& Spruijt-Metz, D. (2006). Associations between physical activity and perceived stress/hassles in college students. Stress and Health, 22, 179-188.

Nogueira-Martins, L. A., Fagnani Neto, R., Macedo, P. C. M., Cítero, V. A., \& Mari, J. J. (2004). The mental health of graduate students at the Federal University of São Paulo: A preliminary report. Brazilian Journal of Medical and Biological Research, 37(10), 1519-1524.

Nyquist, J. D., Manning, L., Wulff, D. H., Austin, A. E., Sprague, J., Fraser, P. K., et al. (1999). On the road to becoming a professor: The graduate student experience. Change: The Magazine of Higher Learning, 31(3), 18-27.

Offstein, E. H., Larson, M. B., McNeill, A. L., \& Mjoni Mwale, H. (2004). Are we doing enough for today's graduate student? International Journal of Educational Management, 18(7), 396-407.

Osiurak, F., Faure, J., Rabeyron, T., Morange, D., Dumet, N., Tapiero, I., et al. (2015). Déterminants de la procrastination académique : motivation autodéterminée, estime de soi et degré de maximation. Pratiques psychologiques, 21(1), 19-33.

Oswalt, S. B., \& Riddock, C. C. (2007). What to do about being overwhelmed: Graduate students, stress and university services. College Student Affairs Journal, 27(1), 24-44.

Pallos, H., Gergely, V., Yamada, N., Miyazaki, S., \& Okawa, M. (2007). The quality of sleep and factors associated with poor sleep in Japanese graduate students. Sleep and Biological Rhythms, 5(4), 234-238.

Parks, A. C. (2013). Positive psychology in higher education. London: Routledge.

Peillod-Book, L., \& Shankland, R. (2016). Manager en pleine conscience. Paris: Dunod.

Peters, B. M. (2007). The relationships among physiological and perceived stress, quality of life, self-care, and impairment in doctoral students. Thèse de doctorat State University of New York at Buffalo.

Powell, K. E., Paluch, A. E., \& Blair, S. N. (2011). Physical activity for health: What kind? How much? How intense? On top of what? Annual Review of Public Health, 32, 349-365.

Rammstedt, B., \& Beierlein, C. (2014). Can't we make it any shorter? The limits of personality assessment and ways to overcome them. Journal of Individual Differences, 35(4), 212-220.

Reise, S. P., Moore, T. M., \& Haviland, M. G. (2010). Bifactor models and rotations: Exploring the extent to which multidimensional data yield univocal scale scores. Journal of Personality Assessment, 92(6), 544-559.

Reynolds, M. J. (2007). Influence of Curriculum on Physical Activity of Athletic Training Graduate Students. Thèse non publiée. Indiana, United States: Indiana State University.

Robins, R. W., Hendin, H. M., \& Trzesniewski, K. H. (2001). Measuring global self-esteem: Construct validation of a single-item measure and the Rosenberg Self-Esteem Scale. Personality and Social Psychology Bulletin, 27(2), 151-161.

Saklofske, D. H., Austin, E. J., Mastoras, S. M., Beaton, L., \& Osborne, S. E. (2012). Relationships of personality, affect, emotional intelligence and coping with student stress and academic success: Different patterns of association for stress and success. Learning \& Individual Differences, 22(2), 251-257.

Saleh, D., Camart, N., \& Romo, L. (2017). Predictors of stress in college students. Frontiers in Psychology, 8 [article 19].

Sasidharan, P., Kolasani, B., \& Divyashanti, C. M. (2016). Prevalence, severity, causes and drugs used for depression, stress and anxiety among junior doctors in a tertiary care teaching hospital in South India. International Journal of Basic and Clinical Pharmacology, 1118-1124.

Schlosser, L. Z., \& Gelso, C. J. (2001). Measuring the working alliance in advisor-advisee relationships in graduate school. Journal of Counseling Psychology, 48(2), 157-167.

Schmidt, M., \& Umans, T. (2014). Experiences of well-being among female doctoral students in Sweden. International Journal of Qualitative Studies on Health and Well-being, 9. http://dx.doi.org/10.3402/qhw.v9.23059

Shah, A. A., Bazargan-Hejazi, S., Lindstrom, R. W., \& Wolf, K. E. (2009). Prevalence of at-risk drinking among a national sample of medical students. Substance Abuse, 30(2), 141-149.

Sjögren, E., Leanderson, P., Kristenson, M., \& Ernerudh, J. (2006). Interleukin-6 levels in relation to psychosocial factors: Studies on serum, saliva, and in vitro production by blood mononuclear cells. Brain, Behavior, and Immunity, 20(3), $270-278$.

Skirka, N. (2000). The relationships of hardiness, sense of coherence, sports participation, and gender to perceived stress and psychological symptoms among college students. Journal of Sports Medicine and Physical Fitness, 40(1), 63-70.

Stecker, T. (2004). Well-being in an academic environment. Medical Education, 38(5), 465-478.

Tavolacci, M. P., Ladner, J., Grigioni, S., Richard, L., Villet, H., \& Dechelotte, P. (2013). Prevalence and association of perceived stress, substance use and behavioral addictions: A cross-sectional study among university students in France, 2009-2011. BMC Public Health, 13, 724-731. 
Taylor, A. H. (2000). Physical activity, anxiety, and stress. Dans S. J. H Biddle, K. R. Fox et S. H. Boutcher (dir.), Physical Activity and Psychological Well-Being (p. 10-45), London and New York: Routledge.

Trafford, V., \& Leshem, S. (2009). Doctorateness as a threshold concept. Innovations in Education and Teaching International, 46(3), 305-316.

Torres, J. B., \& Solberg, V. S. (2001). Role of self-efficacy, stress, social integration, and family support in Latino college student persistence and health. Journal of Vocational Behavior, 59(1), 53-63.

Urbán, R., Kun, B., Farkas, J., Paksi, B., Kökönyei, G., Unoka, Z., et al. (2014). Bifactor structural model of symptom checklists: SCL-90-R and Brief Symptom Inventory (BSI) in a non-clinical community sample. Psychiatry Research, 216(1), 146-154.

Vekkaila, J., Pyhältö, K., \& Lonka, K. (2013). Experiences of disengagement - a study of doctoral students in the behavioral sciences. International Journal of Doctoral Studies, 8, 061-081.

Winwood, P. C., \& Lushington, K. (2006). Disentangling the effects of psychological and physical work demands on sleep, recovery and maladaptive chronic stress outcomes within a large sample of Australian nurses. Journal of Advanced Nursing, 56(6), 679-689.

Wohlgemuth, E., \& Betz, N. (1991). Gender as a moderator of the relationships of stress and social support to physical health in college students. Journal of Counseling Psychology, 38(3), 367-374.

Wright, C. E., Erblich, J., Valdimarsdottir, H. B., \& Bovbjerg, D. H. (2007). Poor sleep the night before an experimental stressor predicts reduced NK cell mobilization and slowed recovery in healthy women. Brain, Behavior \& Immunity, 21(3), 358-363.

Wulf, J. (2010). Relationship among stress, coping, health, and GPA in clinical psychology doctoral students. Thèse de doctorat Chicago School of Professional Psychology.

Zakari, S., Walburg, V., \& Chabrol, H. (2008). Influence de la pression perçue par les lycéens français sur le stress scolaire. Journal de thérapie comportementale et cognitive, 18(3), 108-112.

Zascavage, V., Winterman, K. G., Buot, M., Wies, J. R., \& Lyzinski, N. (2012). Student-life stress in education and health service majors. Higher Education Research \& Development, 31(4), 599-610. 